\title{
PUDA - Privacy and Unforgeability for Data Aggregation
}

\author{
Iraklis Leontiadis, Kaoutar Elkhiyaoui, Melek Önen, Refik Molva \\ EURECOM, Sophia Antipolis, France \\ \{firstname.lastname\}@eurecom.fr
}

\begin{abstract}
Existing work on data collection and analysis for aggregation is mainly focused on confidentiality issues. That is, the untrusted Aggregator learns only the aggregation result without divulging individual data inputs. In this paper we extend the existing models with stronger security requirements. Apart from the privacy requirements with respect to the individual inputs, we ask for unforgeability for the aggregate result. We first define the new security requirements of the model. We also instantiate a protocol for private and unforgeable aggregation for multiple independent users. I.e, multiple unsynchronized users owing to personal sensitive information without interacting with each other, contribute their values in a secure way: The Aggregator learns the result of a function without learning individual values, and moreover, it constructs a proof that is forwarded to a verifier that will convince the latter for the correctness of the computation. Our protocol is provably secure in the random oracle model.
\end{abstract}

\section{Introduction}

With the advent of the Big Data era, research on privacy preserving data collection and analysis is culminating. Users continuously produce data that can be considered as valuable whenever an Aggregator is interested in aggregating users' data. We therefore consider a scenario whereby an Aggregator collects individual data from multiple users who do not interact with each other and executes a function which outputs an aggregate value. This result is further forwarded to the Data Analyzer who can finally extract useful information about the entire population. Various motivating examples under for the aforementioned generic scenario exist in the real-world:

- The analysis of different user profiles and the derivation of statistics can help recommendation engines provide targeted advertisements. In such scenarios a service provider would collect data from each individual user (i.e: on-line purchases), thus acting as an Aggregator, and compute an on-demand aggregate value upon receiving a request from the advertisement company. The latter will further infer some statistics acting as a Data Analyzer, in order to send the appropriate advertisements to each category of users.

- Data aggregation is a promising tool in the field of healthcare research. Different types of data, sensed by body sensors (eg. blood pressure), are collected in large scale in data enclaves who can be considered as Aggregators. Health scientists who act as Data Analyzers are interested in inferring some statistical information from 
these data without having access to each individual input (for privacy and performance reasons). An aggregate value computed over a large population would give very useful information for deriving statistical models, evaluating therapeutic performance or learning the likelihood of upcoming patients' diseases.

Unfortunately, existing solutions only focus on the problem of data confidentiality and consider the Aggregator to be honest-but-curious: the Aggregator is curious in discovering the content of each individual data, but performs the aggregation operation correctly. In this paper we consider a more powerful security model and assume that the Aggregator is untrusted: The Aggregator may provide a bogus aggregate value to the Data Analyzer. In order to protect against such a malicious behavior, we propose that along with the aggregate value, the Aggregator provides a proof of the correctness of the computation of the aggregate result.

The underlying idea of our solution is that each user encrypts its data according to Shi et al. [15] scheme using its own secret encryption key, and sends the resulting ciphertext to the untrusted Aggregator. Users, also homomorphically tag their data using two layers of randomness with two different keys and they forward the tags to the Aggregator. The latter computes the sum by applying operations on the ciphertexts and it also computes a proof for the correctness of the result from the tags. The Aggregator finally sends the result and the proof to the Data Analyzer. The latter verifies the correctness of the computation. We also require the Data Analyzer not to be able to communicate with each user and the result to be publicly verifiable. Moreover, similarly to the existing solutions, the proposed protocol assures obliviousness against the Aggregator and the Data Analyzer in the multi-user setting; meaning that neither the Data Analyzer nor the Aggregator learns individual data inputs.

To the best of our knowledge we are the first to define a model for Privacy and Unforgeability for Data Aggregation (PUDA). We also instantiate a PUDA scheme which mainly pursues the following three objectives:

- Multi-user setting where multiple users produce personal sensitive data without interacting with each other.

- Public verifiability of the aggregate value.

- Privacy of individual data for all participants.

\section{Problem Statement}

We are envisioning a scenario whereby a set of users $\mathbb{U}=\left\{\mathcal{U}_{i}\right\}_{i=1}^{n}$ are producing sensitive data inputs $x_{i, t}$ at each time interval $t$. These individual data are first encrypted into ciphertexts $c_{i, t}$ and further forwarded to an untrusted Aggregator $\mathcal{A}$. Aggregator $\mathcal{A}$ aggregates all the received ciphertexts, decrypts the aggregate and forwards the resulting plaintext to a Data Analyzer $\mathcal{D} \mathcal{A}$ together with a cryptographic proof that assures the correctness of the aggregation operation, which in this paper corresponds to the sum of the users' individual data. An important criterion that we aim to fulfill in this paper is to ensure that Data Analyzer $\mathcal{D} \mathcal{A}$ verifies the correctness of the Aggregator's output without compromising users' privacy. Namely, at the end of the verification operation, both Aggregator $\mathcal{A}$ and Data Analyzer $\mathcal{D} \mathcal{A}$ learn nothing, but the value of the aggregation. 
While homomorphic signatures proposed in $[4,10]$ seem to answer the verifiability requirement, authors in those papers only consider scenarios where a single user generates data.

In the aim of assuring both individual user's privacy and unforgeable aggregation, we first come up with a generic model for privacy preserving and unforgeable aggregation that identifies the algorithms necessary to implement such functionalities and defines the corresponding privacy and security models. Furthermore, we propose a concrete solution which combines an already existing privacy preserving aggregation scheme [15] with an additively homomorphic tag designed for bilinear groups.

Notably, a scheme that allows a malicious Aggregator to compute the sum of users' data in privacy preserving manner and to produce a proof of correct aggregation will start by first running a setup phase. During setup, each user receives a secret key that will be used to encrypt the user's private input and to generate the corresponding authentication tag; the Aggregator $\mathcal{A}$ and the Data Analyzer $\mathcal{D} \mathcal{A}$ on the other hand, are provided with a secret decryption key and a public verification key, respectively. After the key distribution, each user sends its data encrypted and authenticated to Aggregator $\mathcal{A}$, while making sure that the computed ciphertext and the matching authentication tag leak no information about its private input. On receiving users' data, Aggregator $\mathcal{A}$ first aggregates the received ciphertexts and decrypts the sum using its decryption key, then uses the received authentication tags to produce a proof that demonstrates the correctness of the decrypted sum. Finally, Data Analyzer $\mathcal{D} \mathcal{A}$ verifies the correctness of the aggregation, thanks to the public verification key.

\subsection{PUDA Model}

A PUDA scheme consists of the following algorithms:

- Setup $\left(1^{\kappa}\right) \rightarrow\left(\mathcal{P}, \mathrm{SK}_{\mathrm{A}},\left\{\mathrm{SK}_{i}\right\}_{\mathcal{U}_{i} \in \mathbb{U}}, \mathrm{VK}\right)$ : It is a randomized algorithm run by a trusted dealer $\mathcal{K D}$, which on input of a security parameter $\kappa$ outputs the public parameters $\mathcal{P}$ that will be used by subsequent algorithms, the Aggregator $\mathcal{A}$ 's secret key $\mathrm{SK}_{\mathrm{A}}$, the secret keys $\mathrm{SK}_{\mathrm{i}}$ of users $\mathcal{U}_{i}$ and the public verification key VK.

- EncTag $\left(t, \mathrm{SK}_{i}, x_{i, t}\right) \rightarrow\left(c_{i, t}, \sigma_{i, t}\right)$ : It is a randomized algorithm which on inputs of time interval $t$, secret key $\mathrm{SK}_{i}$ of user $\mathcal{U}_{i}$ and data $x_{i, t}$, encrypts $x_{i, t}$ to get a ciphertext $c_{i, t}$ and computes a tag $\sigma_{i, t}$ that authenticates $x_{i, t}$.

- Aggregate $\left(\mathrm{SK}_{\mathrm{A}},\left\{c_{i, t}\right\}_{\mathcal{U}_{i} \in \mathbb{U}},\left\{\sigma_{i, t}\right\}_{\mathcal{U}_{i} \in \mathbb{U}}\right) \rightarrow\left(\operatorname{sum}_{\mathrm{t}}, \sigma_{\mathrm{t}}\right)$ : It is a deterministic algorithm run by the Aggregator $\mathcal{A}$. It takes as inputs Aggregator $\mathcal{A}$ 's secret key $\mathrm{SK}_{\mathrm{A}}$, ciphertexts $\left\{c_{i, t}\right\}_{\mathcal{U}_{i} \in \mathbb{U}}$ and authentication tags $\left\{\sigma_{i, t}\right\}_{\mathcal{U}_{i} \in \mathbb{U}}$, and outputs in cleartext


the correctness of sum $\mathrm{t}_{\mathrm{t}}$, using the authentication tags $\left\{\sigma_{i, t}\right\}_{\mathcal{U}_{i} \in \mathbb{U}}$.

- Verify $\left(\mathrm{VK}, \operatorname{sum}_{\mathrm{t}}, \sigma_{\mathrm{t}}\right) \rightarrow\{0,1\}:$ It is a deterministic algorithm that is executed by the Data Analyzer $\mathcal{D} \mathcal{A}$. It outputs 1 if Data Analyzer $\mathcal{D} \mathcal{A}$ is convinced that the sum $\operatorname{sum}_{\mathrm{t}}=\sum_{\mathcal{U}_{i} \in \mathbb{U}}\left\{x_{i, t}\right\} ;$ and 0 otherwise.

\subsection{Security Model}

In this paper, we only focus on the adversarial behavior of Aggregator $\mathcal{A}$. The rationale behind this is that Aggregator $\mathcal{A}$ is the only party in the protocol that sees all the 
messages exchanged during the protocol execution: Namely, Aggregator $\mathcal{A}$ has access to users' ciphertexts and it is the party that interacts directly with the Data Analyzer. It follows that by ensuring security properties against the Aggregator, one by the same token, ensures these security properties against both Data Analyzer $\mathcal{D} \mathcal{A}$ and external parties.

In accordance with previous work $[11,15]$, we formalize the property of Aggregator obliviousness, which ensures that at the end of a protocol execution, Aggregator $\mathcal{A}$ only learns the sum of users' inputs $x_{i, t}$ and nothing else. Also, we enhance the security definitions of data aggregation with the notion of aggregate unforgeability. As the name implies, aggregate unforgeability guarantees that Aggregator $\mathcal{A}$ cannot forge a valid proof $\sigma_{\mathrm{t}}$ for a sum sum $\mathrm{t}_{\mathrm{t}}$ that was not computed correctly from users' inputs (i.e. cannot generate a proof for $\operatorname{sum}_{\mathrm{t}} \neq \sum x_{i, t}$ ).

Aggregator Obliviousness Aggregator obliviousness ensures that when users $\mathcal{U}_{i}$ provide Aggregator $\mathcal{A}$ with ciphertexts $c_{i, t}$ and authentication tags $\sigma_{i, t}$, Aggregator $\mathcal{A}$ cannot reveal any information about individual inputs $x_{i, t}$, other than the sum value $\sum x_{i, t}$. We extend the existing definition of Aggregator Obliviousness (cf. [11, 12, 15]) so as to capture the fact that Aggregator $\mathcal{A}$ not only has access to ciphertexts $c_{i, t}$, but also has access to the authentication tags $\sigma_{i, t}$ that enable Aggregator $\mathcal{A}$ to generate proofs of correct aggregation.

Similarly to the work of $[11,15]$, we formalize Aggregator obliviousness using an indistinguishability-based game in which Aggregator $\mathcal{A}$ accesses the following oracles:

- $\mathcal{O}_{\text {Setup }}:$ When called by Aggregator $\mathcal{A}$, this oracle initializes the system parameters; it then gives the public parameters $\mathcal{P}$, the Aggregator's secret key $\mathrm{SK}_{\mathrm{A}}$ and public verification key VK to $\mathcal{A}$.

- $\mathcal{O}_{\text {Corrupt }}:$ When queried by Aggregator $\mathcal{A}$ with a user $\mathcal{U}_{i}{ }^{\prime}$ s identifier uid ${ }_{i}$, this oracle provides Aggregator $\mathcal{A}$ with $\mathcal{U}_{i}$ 's secret key denoted $\mathrm{SK}_{\mathrm{i}}$.

- $\mathcal{O}_{\text {EncTag: }}$ : When queried with time $t$, user $\mathcal{U}_{i}$ 's identifier uid ${ }_{i}$ and a data point $x_{i, t}$, this oracle outputs the ciphertext $c_{i, t}$ and the authentication tag $\sigma_{i, t}$ of $x_{i, t}$ computed using $\mathcal{U}_{i}$ 's secret key $\mathrm{SK}_{i}$.

- $\mathcal{O}_{\mathrm{AO}}$ : When called with a subset of users $\mathbb{S} \subset \mathbb{U}$ and with two time-series $\left(\mathcal{U}_{i}, t, x_{i, t}^{0}\right) \mathcal{U}_{i} \in \mathbb{S}$ and $\left(\mathcal{U}_{i}, t, x_{i, t}^{1}\right) \mathcal{U}_{i} \in \mathbb{S}$ such that $\sum x_{i, t}^{0}=\sum x_{i, t}^{1}$, this oracle flips a random coin $b \in\{0,1\}$ and returns an encryption of the time-serie $\left(\mathcal{U}_{i}, t, x_{i, t}^{b}\right) \mathcal{U}_{i} \in \mathbb{S}$ (that is the tuple of ciphertexts $\left\{c_{i, t}^{b}\right\} \mathcal{U}_{i} \in \mathbb{S}$ ) and the corresponding authentication tags $\left\{\sigma_{i, t}^{b}\right\}_{\mathcal{U}_{i} \in \mathbb{S} \text {. }}$

Aggregator $\mathcal{A}$ is accessing the aforementioned oracles during a learning phase (cf. Algorithm 1) and a challenge phase (cf. Algorithm 2). In the learning phase, $\mathcal{A}$ calls oracle $\mathcal{O}_{\text {Setup }}$ which in turn returns the public parameters $\mathcal{P}$, the public verification key $\mathrm{VK}$ and the Aggregator's secret key $\mathrm{SK}_{\mathrm{A}}$. It also interacts with oracle $\mathcal{O}_{\text {Corrupt }}$ to learn the secret keys $\mathrm{SK}_{i}$ of users $\mathcal{U}_{i}$, and oracle $\mathcal{O}_{\text {EncTag }}$ to get a set of ciphertexts $c_{i, t}$ and authentication tags $\sigma_{i, t}$.

In the challenge phase, Aggregator $\mathcal{A}$ chooses a subset $\mathbb{S}^{*}$ of users that were not corrupted in the learning phase, and a challenge time interval $t^{*}$ for which it did not make an encryption query. Oracle $\mathcal{O}_{\mathrm{AO}}$ then receives two time-series $\mathcal{X}_{t^{*}}^{0}=$ 
$\left(\mathcal{U}_{i}, t^{*}, x_{i, t^{*}}^{0}\right)_{\mathcal{U}_{i} \in \mathbb{S}^{*}}$ and $\mathcal{X}_{t^{*}}^{1}=\left(\mathcal{U}_{i}, t^{*}, x_{i, t^{*}}^{1}\right)_{\mathcal{U}_{i} \in \mathbb{S}^{*}}$ from $\mathcal{A}$, such that $\sum x_{i, t^{*}}^{0}=$ $\sum_{\mathcal{U}_{i} \in \mathbb{S}^{*}} x_{i, t^{*}}^{1}$. Then oracle $\mathcal{O}_{\text {AO }}$ flips a random coin $b \stackrel{\$}{\leftarrow}\{0,1\}$ and returns to $\mathcal{A}$ the ciphertexts $\left\{c_{i, t^{*}}^{b}\right\} \mathcal{U}_{i} \in \mathbb{S}^{*}$ and the matching authentication tags $\left\{\sigma_{i, t^{*}}^{b}\right\}_{\mathcal{U}_{i} \in \mathbb{S}^{*}}$.

At the end of the challenge phase, Aggregator $\mathcal{A}$ outputs a guess $b^{*}$ for the bit $b$.

We say that Aggregator $\mathcal{A}$ succeeds in the Aggregator obliviousness game, if its guess $b^{*}$ equals $b$.


Definition 1 (Aggregator Obliviousness). Let $\operatorname{Pr}\left[\mathcal{A}^{\mathrm{AO}}\right]$ denote the probability that Aggregator $\mathcal{A}$ outputs $b^{*}=b$. Then an aggregation protocol is said to ensure Aggregator obliviousness if for any polynomially bounded Aggregator $\mathcal{A}$ the probability $\operatorname{Pr}\left[\mathcal{A}^{\mathrm{AO}}\right] \leqslant \frac{1}{2}+\epsilon(\kappa)$, where $\epsilon$ is a negligible function and $\kappa$ is the security parameter.

Aggregate Unforgeability We augment the security requirements of data aggregation with the requirement of aggregate unforgeability. More precisely, we assume that Aggregator $\mathcal{A}$ is not only interested in compromising the privacy of users participating in the data aggregation protocol, but also interested in tampering with the sum of users' inputs. That is, Aggregator $\mathcal{A}$ may sometimes have an incentive to feed Data Analyzer $\mathcal{D} \mathcal{A}$ erroneous sums. Along these lines, we define aggregate unforgeability as the security feature that ensures that Aggregator $\mathcal{A}$ cannot convince Data Analyzer $\mathcal{D} \mathcal{A}$ to accept a bogus sum, as long as users $\mathcal{U}_{i}$ in the system are honest (i.e. they always submit their correct input and do not collude with the Aggregator $\mathcal{A}$ ).

In compliance with previous work $[7,10]$ on homomorphic signatures, we formalize aggregate unforgeability via a game in which Aggregator $\mathcal{A}$ accesses oracles $\mathcal{O}_{\text {Setup }}$ and $\mathcal{O}_{\text {EncTag. }}$. Furthermore, given the property that anyone holding the public verification key VK can execute the algorithm Verify, we assume that Aggregator $\mathcal{A}$ during the unforgeability game runs the algorithm Verify by itself.

As shown in Algorithm 3, Aggregator $\mathcal{A}$ enters the aggregate unforgeability game by querying the oracle $\mathcal{O}_{\text {Setup }}$ with a security parameter $\kappa$. Oracle $\mathcal{O}_{\text {Setup }}$ accordingly 

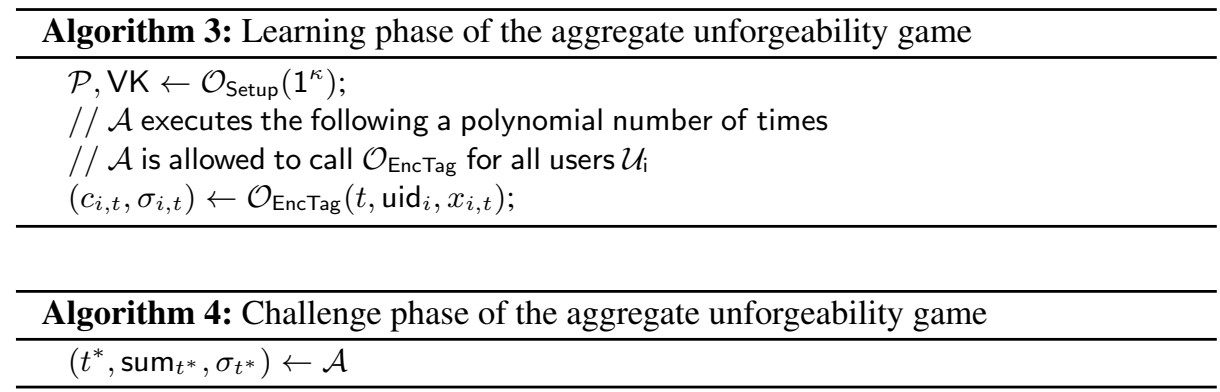

returns public parameters $\mathcal{P}$, verification key VK and the secret key $\mathrm{SK}_{\mathrm{A}}$ of Aggregator $\mathcal{A}$. Moreover, Aggregator $\mathcal{A}$ calls oracle $\mathcal{O}_{\text {EncTag }}$ with tuples $\left(t\right.$, uid $\left._{i}, x_{i, t}\right)$ in order to receive the ciphertext $c_{i, t}$ encrypting $x_{i, t}$ and the matching authenticating tag $\sigma_{i, t}$, both computed using user $\mathcal{U}_{i}$ 's secret key $\mathrm{SK}_{i}$. Note that for each time interval $t$, Aggregator $\mathcal{A}$ is allowed to query oracle $\mathcal{O}_{\text {EncTag }}$ for user $\mathcal{U}_{i}$ only once. In other words, Aggregator $\mathcal{A}$ cannot submit two distinct queries to oracle $\mathcal{O}_{\text {EncTag }}$ with the same time interval $t$ and the same user identifier uid ${ }_{i}$. Without loss of generality, we suppose that for each time interval $t$, Aggregator $\mathcal{A}$ invokes oracle $\mathcal{O}_{\text {EncTag }}$ for all users $\mathcal{U}_{i}$ in the system.

At the end of the aggregate unforgeability game (see Algorithm 4), Aggregator $\mathcal{A}$ outputs a tuple $\left(t^{*}, \operatorname{sum}_{t^{*}}, \sigma_{t^{*}}\right)$. We say that Aggregator $\mathcal{A}$ wins the aggregate unforgeability game if one of the following statements holds:

1. $\operatorname{Verify}\left(\mathrm{VK}, \operatorname{sum}_{t^{*}}, \sigma_{t^{*}}\right) \rightarrow 1$ and Aggregator $\mathcal{A}$ never made a query to oracle $\mathcal{O}_{\text {EncTag }}$ that comprises time interval $t^{*}$. In the remainder of this paper, we denote this type of forgery Type I Forgery.

2. Verify $\left(\mathrm{VK}, \operatorname{sum}_{t^{*}}, \sigma_{t^{*}}\right) \rightarrow 1$ and Aggregator $\mathcal{A}$ has made a query to oracle $\mathcal{O}_{\text {EncTag }}$ for time $t^{*}$, however the sum sum $t^{*} \neq \sum_{\mathcal{U}_{i}} x_{i, t^{*}}$. In what follows, we call this type of forgery Type II Forgery.

Definition 2 (Aggregate Unforgeability). Let $\operatorname{Pr}\left[\mathcal{A}^{\mathrm{AU}}\right]$ denote the probability that Aggregator $\mathcal{A}$ wins the aggregate unforgeability game, that is, the probability that $A g$ gregator $\mathcal{A}$ outputs a Type I Forgery or Type II Forgery that will be accepted by algorithm Verify.

An aggregation protocol is said to ensure aggregate unforgeability if for any polynomially bounded adversary $\mathcal{A}, \operatorname{Pr}\left[\mathcal{A}^{\mathrm{AU}}\right] \leqslant \epsilon(\kappa)$, where $\epsilon$ is a negligible function in the security parameter $\kappa$.

\section{Idea of our PUDA protocol}

In an extended model with an untrusted Aggregator, it is of utmost importance to design a solution in which the untrusted Aggregator cannot provide bogus results to the Data Analyzer. Such a solution will use a proof system that enables the Data Analyzer to verify the correctness of the computation. Yet verifiability should be achieved without 
sacrificing privacy. Towards this goal, we propose a protocol that relies on the following techniques:

- A homomorphic encryption algorithm that allows the Aggregator to compute the sum without divulging individual data.

- A homomorphic tag that allows each user to authenticate the data input $x_{i, t}$, in such a way that the Aggregator can use the collected tags to construct a proof that demonstrates to the Data Analyzer $\mathcal{D} \mathcal{A}$ the correctness of the Aggregator sum.

Concisely, a set of non-interacting users are connected to personal services and devices that produce personal data. Without any coordination, each user chooses a random tag key $t k_{i}$ and sends an encoding thereof, $\overline{t_{k}}$ to the key dealer. After collecting all encoded keys $\overline{\mathrm{tk}_{\mathrm{i}}}$ by users, the key dealer publishes the public verification key VK of this group of users. This verification key is computed as a function of the encodings $\overline{\mathrm{tk}_{\mathrm{i}}}$. Later, the key dealer gives to each user in the system an encryption key ek $k_{i}$ that will be used to compute the user's ciphertexts. Accordingly, the secret key of each user $\mathrm{SK}_{i}$ is defined as the pair of tag key $\mathrm{tk}_{i}$ and encryption key ek ${ }_{i}$. Finally, the key dealer provides the Aggregator with secret key $\mathrm{SK}_{A}$ computed as the sum of encryption keys $\mathrm{ek}_{i}$ and goes off-line.

Now at each time interval $t$, each user employs its secret key $\mathrm{SK}_{\mathrm{i}}$ to compute a ciphertext based on the encryption algorithm of Shi et al. [15] and a homomorphic tag on its sensitive data input. When the Aggregator collects the ciphertexts and the tags

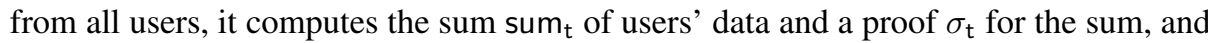
forwards the sum and the proof to the Data Analyzer. At the final step of the protocol, the Data Analyzer verifies with the verification key VK and proof $\sigma_{\mathrm{t}}$ the validity of the

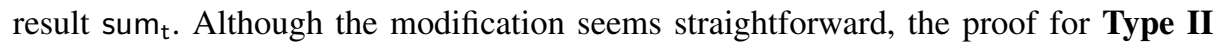
Forgery turns out to be challenging.

Thanks to the homomorphic encryption algorithm of Shi et al. [15] and the way in which we construct our homomorphic tags, we show that our protocol ensures Aggregator obliviousness. Moreover, we show that the Aggregator cannot forge bogus results. Finally, we note that the Data Analyzer $\mathcal{D} \mathcal{A}$ does not keep any state with respect to users' transcripts be they ciphertexts or tags, but it only holds the public verification key, the sum sum $\mathrm{t}$ and the proof $\sigma_{\mathrm{t}}$.

\section{PUDA Instantiation}

Let $\mathbb{G}_{1}, \mathbb{G}_{2}, \mathbb{G}_{T}$ be cyclic groups of large prime order $p$ and $g_{1}, g_{2}$ generators of $\mathbb{G}_{1}, \mathbb{G}_{2}$ accordingly. We say that $e$ is a bilinear map, if the following properties are satisfied:

1. bilinearity: $e\left(g_{1}^{a}, g_{2}^{b}\right)=e\left(g_{1}, g_{2}\right)^{a b}$, where $g_{1}, g_{2} \in \mathbb{G}_{1} \times \mathbb{G}_{2}$ and $a, b \in \mathbb{Z}_{p}$.

2. Computability: there exists an efficient algorithm that computes $e\left(g_{1}^{a}, g_{2}^{b}\right)$ where $g_{1}, g_{2} \in \mathbb{G}_{1} \times \mathbb{G}_{2}$ and $a, b \in \mathbb{Z}_{p}$.

3. Non-degeneracy: $e\left(g_{1}, g_{2}\right) \neq 1$.

For encryption and sum computation we employ the discrete logarithm based encryption scheme of Shi et al. [15]: 


\subsection{Shi-Chan-Rieffel-Chow-Song Scheme}

- Setup $\left(1^{\kappa}\right)$ : Let $\mathbb{G}_{1}$ be a group of large prime order $p$. A trusted key dealer $\mathcal{K D}$ selects a hash function $H:\{0,1\}^{*} \rightarrow \mathbb{G}_{1}$. Furthermore, $\mathcal{K D}$ selects secret encryption keys ek $k_{i} \in \mathbb{Z}_{p}$, uniformly at random. $\mathcal{K D}$ distributes to each user $\mathcal{U}_{i}$ the secret key ek ${ }_{i}$ and it also sends the secret key sk $\mathrm{k}_{\mathrm{A}}=-\sum_{i=1}^{n} \mathrm{ek}_{i}$ to the Aggregator.

- Encrypt $\left(\mathrm{ek}_{i}, x_{i, t}\right)$ : Each user $\mathcal{U}_{i}$ encrypts the value $x_{i, t}$ by using its secret encryption key ek ${ }_{i}$ and outputs the corresponding ciphertext $c_{i, t}=H(t)^{\mathrm{e} k_{i}} g_{1}^{x_{i, t}} \in \mathbb{G}_{1}$.

- Aggregate $\left(\left\{c_{i, t}\right\}_{\mathcal{U}_{i} \in \mathbb{U}},\left\{\sigma_{i, t}\right\}_{\mathcal{U}_{i} \in \mathbb{U}}, \mathrm{SK}_{\mathrm{A}}\right):$ Upon receiving all the ciphertexts $\left\{c_{i, t}\right\}_{i=1}^{n}$, the Aggregator computes: $V_{t}=\left(\prod_{i=1}^{n} c_{i, t}\right) H(t)^{\text {sk }_{A}}=$ $H(t)^{\sum_{i=1}^{n} \mathrm{ek}_{i}} g_{1}^{\sum_{i=1}^{n} x_{i, t}} H(t)^{-\sum_{i=1}^{n} \mathrm{e} \mathrm{k}_{i}}=g_{1}^{\sum_{i=1}^{n} x_{i, t}} \in \mathbb{G}_{1}$. Finally $\mathcal{A}$ learns the sum sum $\sin _{\mathrm{t}}=\sum_{i=1}^{n} x_{i, t} \in \mathbb{Z}_{p}$ by computing the discrete logarithm of $V_{t}$ on the base $g_{1}$. The sum computation is correct as long as $\sum_{i=1}^{n} x_{i, t}<p$.

\subsection{PUDA Scheme}

In what follows we describe our PUDA protocol:

- Setup $\left(1^{\kappa}\right): \mathcal{K} \mathcal{D}$ outputs $\left(p, g_{1}, g_{2}, \mathbb{G}_{1}, \mathbb{G}_{2}, \mathbb{G}_{T}\right)$ for an efficient computable bilinear map $e: G_{1} \times G_{2} \rightarrow G_{T}$, where $g_{1}$ and $g_{2}$ are two random generators for the multiplicative groups $\mathbb{G}_{1}$ and $\mathbb{G}_{2}$ respectively and $p$ is a prime number that denotes the order of all the groups $\mathbb{G}_{1}, \mathbb{G}_{2}$ and $\mathbb{G}_{T}$. Moreover a secret key $a$ is selected by $\mathcal{K D}$. Each $\mathcal{U}_{i}$ selects a random tag key $\mathrm{tk}_{i} \in \mathbb{Z}_{p}$ independently and forwards $g_{2}^{t \mathrm{k}_{i}}$ to $\mathcal{K D}$. $\mathcal{K D}$ publishes the verification key $\mathrm{VK}=\left(\mathrm{vk}_{1}, \mathrm{vk}_{2}\right)=\left(g_{2}^{\sum_{i=1}^{n} \mathrm{tk}_{i}}, g_{2}^{a}\right)$ and distributes to each user $\mathcal{U}_{i} \in \mathbb{U}$ the secret key $g_{1}^{a} \in \mathbb{G}_{1}$ through a secure channel. Thus the secret keys of the scheme are $\mathrm{SK}_{i}=\left(\mathrm{ek}_{i}, \mathrm{tk}_{i}, g_{1}^{a}\right)$. After publishing the public parameters $\mathcal{P}=\left(H, p, g_{1}, g_{2}, \mathbb{G}_{1}, \mathbb{G}_{2}, \mathbb{G}_{T}\right)$ and the verification key VK, $\mathcal{K D}$ goes off-line and it does not further participate in any protocol phase.

- $\operatorname{EncTag}\left(t, \mathrm{SK}_{i}=\left(\mathrm{ek}_{i}, \mathrm{tk}_{i}, g_{1}^{a}\right), x_{i, t}\right)$ : At each time interval $t$ each user $\mathcal{U}_{i}$ encrypts the data value $x_{i, t}$ with its secret encryption key ek ${ }_{i}$, using the encryption algorithm, described in section 4.1, which results in a ciphertext

$$
c_{i, t}=H(t)^{\mathrm{ek}_{i}} g_{1}^{x_{i, t}} \in \mathbb{G}_{1}
$$

$\mathcal{U}_{i}$ also constructs a $\operatorname{tag} \sigma_{i, t} \in \mathbb{G}_{1}$ with its secret tag key $\left(\mathrm{tk}_{i}, g_{1}^{a}\right)$ :

$$
\sigma_{i, t}=H(t)^{\mathrm{tk}_{i}}\left(g_{1}^{a}\right)^{x_{i, t}} \in \mathbb{G}_{1}
$$

Finally $\mathcal{U}_{i}$ sends $\left(c_{i, t}, \sigma_{i, t}\right)$ to $\mathcal{A}$.

- Aggregate $\left(\mathrm{SK}_{\mathrm{A}},\left\{c_{i, t}\right\} \mathcal{U}_{i} \in \mathbb{U},\left\{\sigma_{i, t}\right\}_{\mathcal{U}_{i} \in \mathbb{U}}\right)$ : Aggregator $\mathcal{A}$ computes the sum sum $_{\mathrm{t}}=\sum_{i=1}^{n} x_{i, t}$ by using the Aggregate algorithm presented in section 4.1.

Moreover, $\mathcal{A}$ aggregates the corresponding tags as follows:

$$
\sigma_{\mathrm{t}}=\prod_{i=1}^{n} \sigma_{i, t}=\prod_{i=1}^{n} H(t)^{\mathrm{tk}_{i}}\left(g_{1}^{a}\right)^{x_{i, t}}=H(t)^{\sum \mathrm{t} \mathrm{k}_{i}}\left(g_{1}^{a}\right)^{\sum x_{i, t}}
$$

$\mathcal{A}$ finally forwards sum $\mathrm{t}_{\mathrm{t}}$ and $\sigma_{\mathrm{t}}$ to data analyzer $\mathcal{D} \mathcal{A}$. 
- Verify $\left(\mathrm{VK}, \operatorname{sum}_{\mathrm{t}}, \sigma_{\mathrm{t}}\right)$ : During the verification phase $\mathcal{D} \mathcal{A}$ verifies the correctness of the computation with the verification key $\mathrm{VK}=\left(\mathrm{vk}_{1}=g_{2}^{\sum \mathrm{tk}_{i}}, \mathrm{vk}_{2}=g_{2}^{a}\right)$, by checking the following equality:

$$
e\left(\sigma_{\mathrm{t}}, g_{2}\right) \stackrel{?}{=} e\left(H(t), \mathrm{vk}_{1}\right) e\left(g_{1}^{\mathrm{sum}_{\mathrm{t}}}, \mathrm{vk}_{2}\right)
$$

Verification correctness follows from bilinear pairing properties:

$$
\begin{array}{r}
e\left(\sigma_{\mathrm{t}}, g_{2}\right)=e\left(\prod_{i=1}^{n} \sigma_{i, t}, g_{2}\right)=e\left(\prod_{i=1}^{n} H(t)^{\mathrm{tk}_{i}} g_{1}^{a x_{i, t}}, g_{2}\right)= \\
e\left(H(t)^{\sum_{i=1}^{n} \mathrm{tk}_{i}} g_{1}^{a \sum_{i=1}^{n} x_{i, t}}, g_{2}\right)=e\left(H(t)^{\sum_{i=1}^{n} \mathrm{t} \mathrm{t}_{i}}, g_{2}\right) e\left(g_{1}^{a \sum_{i=1}^{n} x_{i, t}}, g_{2}\right)= \\
e\left(H(t), g_{2}^{\sum_{i=1}^{n} \mathrm{t} \mathrm{k}_{i}}\right) e\left(g_{1}^{\sum_{i=1}^{n} x_{i, t}}, g_{2}^{a}\right)= \\
e\left(H(t), g_{2}^{\sum_{i=1}^{n} \mathrm{t} \mathrm{k}_{i}}\right) e\left(g_{1}^{\mathrm{sum}_{\mathrm{t}}}, g_{2}^{a}\right)=e\left(H(t), \mathrm{vk}_{1}\right) e\left(g_{1}^{\mathrm{sum}_{\mathrm{t}}}, \mathbf{v k}_{2}\right)
\end{array}
$$

\section{Analysis}

\subsection{Aggregator Obliviousness}

Theorem 1. The proposed solution achieves aggregator obliviousness in the random oracle model under the decisional Diffie-Hellman $(D D H)$ assumption in $\mathbb{G}_{1}$.

Proof. Assume there is an aggregator $\mathcal{A}$ which breaks the obliviousness of the PUDA scheme with a non-negligible advantage $\epsilon$. We build in what follows an adversary $\mathcal{B}$ who uses $\mathcal{A}$ as a subroutine to break the aggregator obliviousness of the private streaming aggregation (PSA) protocol presented in [15], which is guaranteed under DDH. Without loss of generality we call the oracles that the adversary $\mathcal{B}$ has access to from the PSA scheme as follows: $\mathcal{O}_{\text {Setup }}^{\text {PSA }}, \mathcal{O}_{\text {Corrupt }}^{\text {PSA }}, \mathcal{O}_{\text {Encrypt }}^{\text {PSA }}$, and $\mathcal{O}_{\mathrm{AO}}^{\text {PSA }}$.

We consider in PSA as in PUDA that there are $n$ users $\mathcal{U}_{i}$ and each one of these users possesses a secret encryption key $\mathrm{ek}_{\mathbf{i}}$. In the following, we show how an adversary $\mathcal{B}$ simulates the aggregator obliviousness game presented in Algorithms 1 and 2 to aggregator $\mathcal{A}$ and how therewith breaks the aggregator obliviousness of PSA.

Learning phase: In the learning phase, adversary $\mathcal{B}$ proceeds as following: Whenever $\mathcal{A}$ calls oracle $\mathcal{O}_{\text {Setup }}$ with a security parameter $\kappa, \mathcal{B}$ queries oracle $\mathcal{O}_{\text {Setup }}^{\text {PSA }}$ with the same security parameter. Oracle $\mathcal{O}_{\text {Setup }}^{\text {PSA }}$ in turn outputs the public parameters that are composed of a hash function $H:\{0,1\}^{*} \rightarrow \mathbb{G}_{1}$, a generator $g_{1}$ of the group $\mathbb{G}_{1}$ of safe prime order $p$, and the aggregator's secret key $\mathrm{SK}_{\mathrm{A}}=-\sum_{\mathrm{i}=1}^{\mathrm{n}} \mathrm{e \textrm {k } _ { \mathrm { i } }} \cdot \mathcal{B}$ then selects the parameters of a bilinear pairing $\left(e, g_{1}, g_{2}, \mathbb{G}_{1}, \mathbb{G}_{2}, \mathbb{G}_{T}\right) \cdot \mathcal{B}$ chooses uniformly at random $a,\left\{r_{i}\right\}_{\mathcal{U}_{i} \in \mathcal{U}}$ such and defines the verification key $\mathrm{VK}$ as follows:

$$
\mathrm{VK}=\left(g_{2}^{a S K_{\mathrm{A}}+\sum_{i=1}^{n} r_{i}}, g_{2}^{a}\right)=\left(g_{2}^{a \sum_{i=1}^{n} \mathrm{e} \mathrm{k}_{i}+\sum_{i=1}^{n} r_{i}}, g_{2}^{a}\right)=\left(g_{2}^{\sum_{i=1}^{n} a \mathrm{ek} i+r_{i}}, g_{2}^{a}\right)
$$

This entails that $\mathrm{tk}_{i}$ is defined as: $a \mathrm{ek}_{i}+r_{i}$. Finally $\mathcal{B}$ forwards to $\mathcal{A}$ the public parameters: $\mathcal{P}=\left(H, p, g_{1}, g_{2}, \mathbb{G}_{1}, \mathbb{G}_{2}, \mathbb{G}_{T}\right)$, the verification keys $\mathrm{VK}=\left(g_{2}^{\sum_{i=1}^{n} \mathrm{t}_{\mathrm{i}}}, g_{2}^{a}\right)$ and the secret key of the Aggregator sk $\mathrm{A}$. 
Whenever $\mathcal{A}$ calls oracle $\mathcal{O}_{\text {Corrupt }}$ with a user's identifier uid ${ }_{i}, \mathcal{B}$ relays the query uid $_{i}$ to $\mathcal{O}_{\text {Corrupt }}^{\text {PSA }}$ of the PSA scheme which in turns outputs the secret encryption key ek $k_{i}$ of user $\mathcal{U}_{i} . \mathcal{B}$ then returns secret key $\mathrm{SK}_{i}=\left(\mathrm{ek}_{i}, \mathrm{tk}_{i}\right)=\left(\mathrm{ek}_{i}, a \mathrm{ek}_{i}+r_{i}\right)$.

Whenever $\mathcal{A}$ calls oracle $\mathcal{O}_{\text {EncTag }}$ with query $\left(t\right.$, uid $\left._{i}, x_{i, t}\right), \mathcal{B}$ forwards the query to the $\mathcal{O}_{\text {Encrypt }}^{\text {PSA }}$ oracle which returns the appropriate ciphertext $c_{i, t}=H(t)^{\text {ek }} g_{1}^{x_{i, t}} . \mathcal{B}$ computes then the tag associated with ciphertext $c_{i, t}$ as $\sigma_{i, t}=\left(c_{i, t}\right)^{a} H(t)^{r_{i}}=H(t)^{a e \mathrm{k}_{\mathrm{i}}+\mathrm{r}_{\mathrm{i}}} g_{1}^{a x_{i, t}}=H(t)^{\mathrm{t} \mathrm{k}_{i}} g_{1}^{a x_{i, t}}$ and transmits to $\mathcal{A}$ ciphertext $c_{i, t}$ and tag $\sigma_{i, t}$.

Challenge phase: In the challenge phase $\mathcal{A}$ chooses a set of users $\mathbb{S}^{*}$ that have not been corrupted during the learning phase and a time interval $t^{*}$ for which $\mathcal{A}$ did not make a query to oracle $\mathcal{O}_{\text {EncTag. }} \mathcal{A}$ then submits two time-series $\mathcal{X}_{0}^{*}=\left(\mathcal{U}_{i}, t^{*}, x_{i, t^{*}}^{0}\right) \mathcal{U}_{i} \in \mathbb{S}^{*}$ and $\mathcal{X}_{1}^{*}=\left(\mathcal{U}_{i}, t^{*}, x_{i, t^{*}}^{1}\right) \mathcal{U}_{i} \in \mathbb{S}^{*}$ to $\mathcal{O}_{\mathrm{AO}}$, such that $\sum x_{i, t^{*}}^{0}=\sum x_{i, t^{*}}^{1} \cdot \mathcal{B}$ simulates this oracle as follows:

It forwards the series $\mathcal{X}_{0}^{*}$ and $\mathcal{X}_{1}^{*}$ to $\mathcal{O}_{\mathrm{AO}}^{\mathrm{PSA}}$ which chooses uniformly at random a bit $b \stackrel{\&}{\leftarrow}\{0,1\}$ and returns to $\mathcal{B}$ the ciphertexts $\left\{c_{i, t^{*}}^{b}\right\}_{\mathcal{U}_{i} \in \mathbb{S}^{*}}$ encrypting time-serie $\mathcal{X}_{b}^{*}$.

Next, $\mathcal{B}$ constructs for all $\mathcal{U}_{i}$ in $\mathbb{S}^{*}$ the tag $\sigma_{i, t^{*}}^{b}$ corresponding to ciphertext $c_{i, t^{*}}^{b}$ by computing:

$$
\begin{array}{r}
\sigma_{i, t^{*}}^{b}=\left(c_{i, t}^{b}\right)^{a} H\left(t^{*}\right)^{r_{i}}=\left(H\left(t^{*}\right)^{\mathrm{ek}} g_{1}^{x_{i, t^{*}}^{b}}\right)^{a} H\left(t^{*}\right)^{r_{i}} \\
=H\left(t^{*}\right)^{a \mathrm{ek}_{i}+r_{i}} g_{1}^{a x_{i, t^{*}}^{b}}=H\left(t^{*}\right)^{\mathrm{t} \mathrm{t}_{i}} g_{1}^{a x_{i, t^{*}}^{b}}
\end{array}
$$

Note that $\sigma_{i, t^{*}}^{b}$ corresponds to a correctly computed tag for input $x_{i, t^{*}}^{b}$. Finally, $\mathcal{B}$ forwards to $\mathcal{A}\left\{\left(c_{i, t^{*}}^{b}, \sigma_{i, t^{*}}^{b}\right\}_{\mathcal{U}_{i} \in \mathbb{S}^{*}}\right.$. At this point, the simulated view of aggregator $\mathcal{A}$ is computationally indistinguishable from its view in an actual aggregator obliviousness game as defined in Algorithms 1 and 2. This leads to correct verification of the sum computed by $\mathcal{A}$, more precisely:

$$
\begin{array}{r}
e\left(\prod_{i \in \mathbb{S}^{*}} \sigma_{i, t^{*}}^{b}, g_{2}\right)=e\left(\prod_{i=1}^{n} H\left(t^{*}\right)^{\mathrm{tk}_{i}} g_{1}^{a x_{i, t^{*}}^{b}}, g_{2}\right) \\
=e\left(H\left(t^{*}\right), g_{2}^{a \sum_{i=1}^{n} \mathrm{ek} i+\sum_{i=1}^{n} r_{i}}\right) e\left(g_{1}^{\sum_{i=1}^{n} x_{i, t^{*}}^{b}}, g_{2}^{a}\right)=e\left(H\left(t^{*}\right), \mathrm{vk}_{1}\right) e\left(g_{1}^{\sum_{i=1}^{n} x_{i, t^{*}}^{b}}, \mathrm{vk}_{2}\right)
\end{array}
$$

It follows that if aggregator $\mathcal{A}$ is able to output a correct guess $b^{*}$ for the bit $b$ with a non-negligible advantage $\epsilon$ : (i.e. is able to break the aggregator obliviousness of our scheme), then $\mathcal{B}$ will break the aggregator obliviousness of the PSA scheme with the same non-negligible advantage $\epsilon$ by outputting the guess $b^{*}$.

As such PSA scheme ensures aggregator obliviousness under the DDH assumption in $\mathbb{G}_{1}$, we can conclude that our scheme also ensures aggregator obliviousness: $\operatorname{Pr}\left[\mathcal{A}^{\mathbf{A O}}\right] \leqslant \frac{1}{2}+\epsilon(\kappa)$ as long as DDH holds in $\mathbb{G}_{1}$. 


\subsection{Aggregate Unforgeability}

We first introduce a new assumption that is used during the security analysis of our PUDA instantiation. Our new assumption named hereafter as LEOM is a variant of the LRSW assumption [14] which is proven secure in the generic model [16] and it used for the construction of the CL signatures [5]. W.l.g we assume a set $I$ of size $n$ and an index $t$. The $\mathcal{O}_{\text {LEOM }}$ oracle chooses $\left\{\gamma_{i}\right\}_{i=1}^{n}, \forall i \in I, \delta \in \mathbb{Z}_{p}$ uniformly and at random which are kept secret. It also gives the public key $\left(g_{2}^{\sum_{i=1}^{n} \gamma_{i}}, g_{2}^{\delta}\right)$ to the adversary and chooses $\alpha \in \mathbb{G}_{1}$ at random. Adversary makes bulk queries $\left(i, t,\left\{x_{i, t}\right\}_{i=1}^{n}\right), \forall i \in I$ and the $\mathcal{O}_{\text {LEOM }}$ oracle, chooses $\beta_{t} \in \mathbb{Z}_{p}$ uniformly and at random and replies with $\left\{\left(\alpha, \beta_{t}, \beta_{t}^{\gamma_{i}} \alpha^{\delta x_{i, t}}\right)\right\}_{i=1}^{n}$ for each different $t$. $\mathcal{O}_{\text {LEOM }}$ aborts if it receives a bulk query for a $t$ for which there is $i^{\prime} \in I: i=i^{\prime}$ for which $x_{i, t} \neq x_{i, t}^{\prime}$. In the end the adversary succeeds if it outputs a tuple $\left(t, z, \alpha, \beta_{t}, \beta_{t}^{\sum_{i=1}^{n} \gamma_{i}} \alpha^{\delta z}\right)$ for a $t$ in which $\sum_{i=1}^{n} x_{i, t} \neq z$.

Theorem 2. (LEOM Assumption) Let $\mathcal{G}$ be an algorithm that on input the security parameter $\kappa$ outputs the parameters of a bilinear group $\mathrm{G}=\left(e, \mathbb{G}_{1}, \mathbb{G}_{2}, g_{1}, g_{2}, p\right)$. Define $\Delta=g_{2}^{\delta}, \Gamma=g_{2}^{\sum_{i=1}^{n} \gamma_{i}} \in \mathbb{G}_{2}^{2}$ for $\delta, \gamma_{i} \in \mathbb{Z}_{p}, \forall i \in I$. Consider an oracle $\mathcal{O}_{\mathrm{LEOM}}$ that on input a set of queries $\left(i, t,\left\{x_{i, t}\right\}_{i=1}^{n}\right)$ responds with $\left(\alpha, \beta_{t}, \beta_{t}^{\gamma_{i}} \alpha^{x_{i, t} \delta}\right)$ for a uniformly at random element $\alpha \in \mathbb{G}_{1}, \beta_{t} \in \mathbb{Z}_{p}$.

Then for all probabilistic polynomial time adversaries $\mathcal{A}$ the probability:

$$
\begin{aligned}
& \operatorname{Pr}\left[\mathrm{G} \leftarrow \mathcal{G}\left(1^{\kappa}\right) ; \delta, \gamma_{i}\right. \in \mathbb{Z}_{p} ;\left(\Gamma=g_{2}^{\delta}, \Delta=g_{2}^{\sum_{i=1}^{n} \gamma_{i}}\right) ; \\
&(t, z, a, b, c) \leftarrow \mathcal{A}^{\mathcal{O} \text { LEOM }\left(i, t,\left\{x_{i, t}\right\}_{i=1}^{n}\right)}: \\
&\left.\left(z \neq \sum_{i=1}^{n} x_{i, t}, t\right) \wedge a=\alpha \wedge b=\beta_{t} \wedge c=\beta_{t}^{\sum_{i=1}^{n} \gamma_{i}} \alpha^{z \delta}\right] \leq \epsilon_{2}(\kappa)
\end{aligned}
$$

Due to space limitations, the security evidence of the LEOM is deferred in the Appendix section.

We show in our analysis that a Type I Forgery implies a break of the BCDH assumption and next that a Type II Forgery implies a break of the LEOM assumption.

Theorem 3. Our scheme achieves aggregate unforgeability against a Type I Forgery under $\mathrm{BCDH}$ assumption in the random oracle model.

Proof. We show how to build an attacker $\mathcal{B}$ that solves BCDH in $\left(\mathbb{G}_{1}, \mathbb{G}_{2}, \mathbb{G}_{T}\right)$. Let $g_{1}$ and $g_{2}$ be two generators for $\mathbb{G}_{1}$ and $\mathbb{G}_{2}$ respectively. $\mathcal{B}$ receives the challenge $\left(g_{1}, g_{2}, g_{1}^{a}, g_{1}^{b}, g_{1}^{c}, g_{2}^{a}, g_{2}^{b}\right)$ from the BCDH oracle $\mathcal{O}_{\mathrm{BCDH}}$ and is asked to output $e\left(g_{1}, g_{2}\right)^{a b c} \in \mathbb{G}_{T} \cdot \mathcal{B}$ simulates the interaction with $\mathcal{A}$ in the two phases (Setup, Learning) as follows:

Setup:

- To simulate the $\mathcal{O}_{\text {Setup }}^{\mathcal{A}}$ oracle $\mathcal{B}$ selects uniformly at random $2 n$ keys $\left\{\mathrm{k}_{i}\right\}_{i=1}^{n}$, $\left\{\mathrm{y}_{i}\right\}_{i=1}^{n} \in \mathbb{Z}_{p}$ and outputs the public parameters $\mathcal{P}=\left(\kappa, p, g_{1}, g_{2}, \mathbb{G}_{1}, \mathbb{G}_{2}\right)$ the verification key $\mathrm{VK}=\left(\mathrm{vk}_{1}, \mathrm{vk}_{2}\right)=\left(g_{2}^{b \sum_{i=1}^{n} \mathrm{k}_{i}}, g_{2}^{a}\right)$ and the secret key of the Aggregator $\mathrm{SK}_{A}=-\sum_{i=1}^{n} \mathrm{y}_{\mathrm{i}}$. 


\section{Learning phase}

- $\mathcal{A}$ is allowed to query the random oracle $H$ for any time interval . $\mathcal{B}$ constructs a $\mathrm{H}-$ list and responds to $\mathcal{A}$ query as follows:

1. If query $(t)$ already appears in a tuple $H$-tuple $\left\langle t: r_{t}, \operatorname{coin}(t), H(t)\right\rangle$ of the $\mathrm{H}-$ list it responds to $\mathcal{A}$ with $H(t)$.

2. Otherwise it selects a random number $r_{t} \in \mathbb{Z}_{p}$ and flips a random coin $\stackrel{\&}{\leftarrow}\{0,1\}$. With probability $p$, coin $(\mathrm{t})=0$ and $\mathcal{B}$ answers with $H(t)=$ $g_{1}^{r_{t}}$. Otherwise if coin $(\mathrm{t})=1$ then $\mathcal{B}$ responds with $H(t)=g_{1}^{c r_{t}}$ and updates the $\mathrm{H}-$ list with the new tuple $H$-tuple $\left\langle t: r_{t}\right.$, coin $\left.(t), H(t)\right\rangle$.

- Whenever $\mathcal{A}$ submits a query $\left(t\right.$, uid $\left._{i}, x_{i, t}\right)$ to the $\mathcal{O}_{\text {EncTag }}^{\mathcal{A}}, \mathcal{B}$ constructs a T - list and responds as follows:

1. If at time interval $t \mathcal{A}$ has never queried before the $\mathcal{O}_{\text {EncTag }}^{\mathcal{A}}$ oracle then:

(a) $\mathcal{B}$ initializes variable $\Sigma_{t}=0$.

(b) $\mathcal{B}$ calls the simulated random oracle, receives the result for $\mathrm{H}(\mathrm{t})$ and appends the tuple $H$-tuple $\left\langle t: r_{t}\right.$, coin $\left.(t), H(t)\right\rangle$ to the $\mathrm{H}$ - list.

(c) If $\operatorname{coin}(t)=1$ then $\mathcal{B}$ stops the simulation.

(d) Otherwise it chooses the secret tag key $\mathrm{k}_{i}$ where $i=$ uid $_{\mathrm{i}}$ to be used as secret tag key from the set of $\left\{\mathrm{k}_{i}\right\}$ keys, chosen by $\mathcal{B}$ in the Setup phase.

(e) $\mathcal{B}$ sends to $\mathcal{A}$ the tag $\sigma_{i, t}=g_{1}^{r_{r} b k_{i}} g_{1}^{a x_{i, t}}=H(t)^{b k_{i}} g_{1}^{a x_{i, t}}$, which is a valid tag for the value $x_{i, t}$. Notice that $\mathcal{B}$ can correctly compute the tag without knowing $a$ and $b$ from the $\mathrm{BCDH}$ problem parameters $g_{1}^{a}, g_{1}^{b}$.

(f) $\mathcal{B}$ chooses also a secret encryption key $\mathrm{y}_{i} \in\left\{\mathrm{y}_{i}\right\}_{i=1}^{n} \in \mathbb{Z}_{p}$ and computes the ciphertext as $c_{i, t}=H(t)^{\mathrm{y}_{i}} g_{1}^{x_{i, t}}$. The simulation is correct since $\mathcal{A}$ can check that the sum $\sum_{i=1}^{n} x_{i, t}$ corresponds to the ciphertexts given by $\mathcal{B}$ with its decryption key $\mathrm{SK}_{A}=-\sum_{i=1}^{n} \mathrm{y}_{\mathrm{i}}$, considering the attacker has made distinct encryption queries for all the $n$ users in the scheme at a time interval $t$.

(g) $\mathcal{B}$ sets $\Sigma_{t}=\Sigma_{t}+x_{i, t}$ and updates the $\mathrm{T}-$ list with the tuple: $\left\langle t, \operatorname{uid}_{i}, x_{i, t}, \sigma_{i, t}\right\rangle$

2. Else if $\mathrm{T}-1$ ist contains $i^{\prime}=\mathrm{uid}_{\mathrm{i}}$ and $x_{i, t}=x_{i, t}^{\prime}$ then $\mathcal{B}$ fetches the corresponding $\sigma_{i, t}$ from the list and forwards it to $\mathcal{A}$.

3. Else if $\mathrm{T}-$ list contains $i^{\prime}=$ uid and $x_{i, t} \neq x_{i, t}^{\prime}$ then $\mathcal{B}$ aborts.

4. Otherwise $\left(0<\operatorname{cnt}_{\mathrm{t}}<n\right), \mathcal{B}$ looks to the $\mathrm{H}-$ list list for the tuple indexed by $t$ in order to get $\left\langle t: r_{t}, \operatorname{coin}(t), H(t)\right\rangle$. If the tuple does not exist then $\mathcal{B}$ tosses a random coin and if $\operatorname{coin}(t)=1$ then $\mathcal{B}$ aborts. If $\operatorname{coin}(t)=0$ then $\mathcal{B}$ computes the tag identically as in $1(\mathrm{~d})(\mathrm{e})(\mathrm{f})(\mathrm{g})$ steps: It chooses a key $\mathrm{k}_{i}$ where $i=$ uid $_{\mathrm{i}}$ from the selected keys $\left\{\mathrm{k}_{i}\right\}$. It constructs the tag as $\sigma_{i, t}=$ $g_{1}^{r_{t} b k_{i}} g_{1}^{a x_{i, t}}=H(t)^{b k_{i}} g_{1}^{a x_{i, t}}$ and the ciphertext as $c_{i, t}=H(t)^{\mathrm{y}_{i}} g_{1}^{x_{i, t}}$. Finally $\mathcal{B}$ sets $\Sigma_{t}=\Sigma_{t}+x_{i, t}$, updates the T - list with the tuple: $\left\langle t\right.$, uid $\left._{i}, x_{i, t}, \sigma_{i, t}\right\rangle$.

Now, when $\mathcal{B}$ receives the forgery $\left(\operatorname{sum}_{\mathrm{t}}{ }^{*}, \sigma_{\mathrm{t}}{ }^{*}\right)$ at time interval $t=t^{*}$, it continues if sum $_{\mathrm{t}}{ }^{*} \neq \Sigma_{t}$. $\mathcal{B}$ first queries the $H$-tuple for time $t^{*}$ in order to fetch the appropriate tuple.

- If $\operatorname{coin}\left(t^{*}\right)=0$ then $\mathcal{B}$ aborts. 
- If coin $\left(t^{*}\right)=1$ then since $\mathcal{A}$ outputs a valid forged $\sigma_{\mathrm{t}}{ }^{*}$ at $t^{*}$, it is true that the following equation should hold:

$$
e\left(\sigma_{\mathrm{t}}^{*}, g_{2}\right)=e\left(H\left(t^{*}\right), \mathrm{vk}_{1}\right) e\left(g_{1}^{\mathrm{sum}_{\mathrm{t}}^{*}}, \mathrm{vk}_{2}\right)
$$

which is true when $\mathcal{A}$ makes $n$ queries for time interval $t^{*}$ for distinct users to the $\mathcal{O}_{\text {EncTag }}^{\mathcal{A}}$ oracle during the Learning phase. As such $\sigma_{\mathrm{t}}{ }^{*}=g_{1}^{c r_{t} b \sum \mathrm{k}_{i}} g_{1}^{a \text { sum }_{\mathrm{t}}{ }^{*}}$

Finally $\mathcal{B}$ outputs:

$$
\begin{aligned}
& e\left(\left(\frac{\sigma_{\mathrm{t}}^{*}}{g_{1}^{a \text { sum }_{\mathrm{t}}^{*}}}\right)^{\frac{1}{r_{t} \sum \mathrm{k}_{i}}}, g_{2}^{a}\right)=e\left(\left(\frac{g_{1}^{c r_{t} b \sum \mathrm{k}_{i}} g_{1}^{a \text { sum }_{\mathrm{t}}^{*}}}{g_{1}^{a \text { sum }_{\mathrm{t}}^{*}}}\right)^{\frac{1}{r_{t} \sum \mathrm{k}_{i}}}, g_{2}^{a}\right) \\
& \quad=e\left(\left(g_{1}^{c r_{t} b \sum \mathrm{k}_{i}}\right)^{\frac{1}{r_{t} \sum \mathrm{k}_{i}}}, g_{2}^{a}\right)=e\left(g_{1}^{b c}, g_{2}^{a}\right)=e\left(g_{1}, g_{2}\right)^{a b c}
\end{aligned}
$$

Let $\mathcal{A}^{\mathrm{AU} 1}$ the event when $\mathcal{A}$ successfully forges a Type I forgery $\sigma_{\mathrm{t}}$ for our PUDA protocol that happens with some non-negligible probability $\epsilon^{\prime}$. Then $\operatorname{Pr}\left[\mathcal{B}^{\mathrm{BCDH}}\right]=$ $\operatorname{Pr}\left[\right.$ event $\left._{0}\right] \operatorname{Pr}\left[\right.$ event $\left._{1}\right] \operatorname{Pr}\left[\mathcal{A}^{\mathbf{A U 2}}\right]=p(1-p)^{\mathrm{q}_{\mathrm{H}}-1} \epsilon^{\prime}$, for $\mathrm{q}_{\mathrm{H}}$ random oracle queries with the probability $\operatorname{Pr}[\operatorname{coin}(t)=0]=p$. As such we ended up in a contradiction assuming the hardness of the $\mathrm{BCDH}$ assumption and finally $\operatorname{Pr}\left[\mathcal{A}^{\mathrm{AU1}}\right] \leq \epsilon_{1}$, where $\epsilon_{1}$ is a negligible function.

Theorem 4. Our scheme guarantees aggregate unforgeability against a Type II Forgery under the LEOM assumption in the random oracle model.

Proof. (Sketch) The $\mathcal{O}_{\text {EncTag }}^{\mathcal{A}}$ oracle behaves equivalently as the oracle in the LEOM assumption. $\mathcal{B}$ chooses secret encryptions keys $\left\{\mathrm{ek}_{i}\right\}_{i=1}^{n}$ and sends to $\mathcal{A}$ the secret decryption key $\mathrm{SK}_{A}=-\sum_{i=1}^{n} \mathrm{ek}_{i} \cdot \mathcal{B}$ receives also the public key $\left(\mathrm{vk}_{1}=g_{2}^{\sum_{i=1}^{n} \gamma_{i}}, \mathrm{vk}_{2}=g_{2}^{\delta}\right)$ from the $\mathcal{O}_{\text {LEOM }}$ oracle and forwards it to $\mathcal{A}$ along with the public parameters $\mathcal{P}=$ $\left(\kappa, p, g_{1}=\alpha, g_{2}, \mathbb{G}_{1}, \mathbb{G}_{2}\right)$. For a random oracle query $\mathrm{H}(\mathrm{t})$ the simulator $\mathcal{B}$ queries the $\mathcal{O}_{\text {LEOM }}$ with input $\left(i \ni I, t, x_{i, t} \stackrel{\$}{\leftarrow} \mathbb{Z}_{p}\right)$ which replies with $\left(a=\alpha \wedge b=\beta_{t} \wedge c=\right.$ $\left.\beta_{t}^{\gamma_{i}} \alpha^{x_{i, t} \delta}\right)$. Finally $\mathcal{B}$ forwards to $\mathcal{A}, H(t)=\beta_{t}$. For queries $\left(i=\mathrm{uid}, t, x_{i, t}\right)$ to the $\mathcal{O}_{\text {EncTag }}^{\mathcal{A}}$ oracle the simulator $\mathcal{B}$ returns $\sigma_{i, t}=\beta_{t}^{\gamma_{i}} \alpha^{\delta x_{i, t}}$ from the $\mathcal{O}_{\text {LEOM }}$ oracle, as a tag, and constructs the ciphertext as $c_{i, t}=\beta_{t}^{\mathrm{ek}_{i}} g_{1}^{x_{i, t}}$. $\mathcal{A}$ is able to correctly verify the sum, more precisely:

$$
\begin{aligned}
& e\left(\prod_{i=1}^{n} \sigma_{i, t}, g_{2}\right)=e\left(\prod_{i=1}^{n} \beta_{t}^{\gamma_{i}} \alpha^{\delta x_{i, t}}, g_{2}\right)=e\left(\beta_{t}^{\sum_{i=1}^{n} \gamma_{i}} \alpha^{\delta \sum_{i=1}^{n} x_{i, t}}, g_{2}\right) \\
& =e\left(\beta_{t}, g_{2}^{\sum_{i=1}^{n} \gamma_{i}}\right) e\left(\alpha^{\sum_{i=1}^{n} x_{i, t}}, g_{2}^{\delta}\right)=e\left(\beta_{t}, \mathrm{vk}_{1}\right) e\left(\alpha^{\sum_{i=1}^{n} x_{i, t}}, \mathrm{vk}_{2}\right)
\end{aligned}
$$

Therefore, from the point of view of $\mathcal{A}$, the tags $\sigma_{i, t}=\beta_{t}^{\gamma_{i}} \alpha^{\delta x_{i, t}}$ correspond to well formed verifiable tags. Notice that if there is some non-negligible probability that $\mathcal{A}$ outputs a valid Type II Forgery, then $\mathcal{B}$ breaks the LEOM assumption with some nonnegligible probability. This leads to a contradiction under the LEOM assumption and accordingly, $\operatorname{Pr}\left[\mathcal{A}^{\mathbf{A U} \mathbf{2}}\right] \leq \epsilon_{2}$ for a negligible function $\epsilon_{2}$. We conclude that our scheme guarantees aggregate unforgeability for a Type II Forgery under the LEOM assumption in the random oracle model. 


\begin{tabular}{l||c|c|}
\hline Participant & Computation & Communication \\
\hline User & $\mathbf{2} \mathrm{EXP}+\mathbf{1} \mathrm{MUL}$ & $\mathbf{2} \cdot \mathbf{l}$ \\
\hline Aggregator & $(\mathbf{n}-\mathbf{1}) \mathrm{MUL}$ & $\mathbf{2} \cdot \mathbf{l}$ \\
\hline Data Analyzer & $\mathbf{3}$ PAIR $+\mathbf{1} \mathrm{EXP}+\mathbf{1} \mathrm{MUL}+\mathbf{1 H} A S H$ & - \\
\hline
\end{tabular}
$\begin{aligned} & \text { Table 1: Performance of tag computation, proof construction and verification operations. } l \text { denotes the bit-size of the prime } \\
& \text { number } p \text {. }\end{aligned}$

To conclude with the analysis the success probabilities for the aggregate unforgeability game $\operatorname{Pr}\left[\mathcal{A}^{\mathrm{AU}}\right]$, are taken over the union of the success probabilities for the two type of forgeries. As such

$$
\operatorname{Pr}\left[\mathcal{A}^{\mathbf{A U}}\right]=\operatorname{Pr}\left[\mathcal{A}^{\mathbf{A U} \mathbf{1}}\right]+\operatorname{Pr}\left[\mathcal{A}^{\mathbf{A U} \mathbf{2}}\right] \leq \epsilon_{1}(\kappa)+\epsilon_{2}(\kappa)
$$

where $\epsilon_{1}$ and $\epsilon_{2}$ are negligible functions.

\subsection{Performance Evaluation}

In this section we analyze the extra overhead of ensuring the aggregate unforgeability property in our PUDA instantiation scheme. First, we consider a theoretical evaluation with respect to the mathematical operations a participant of the protocol be it user, $\mathrm{Ag}$ gregator or Data Analyzer has to perform with respect to the verifiability transcripts. That is, the computation of the tag by each user, the proof by the Aggregator and the verification of the proof by the Data Analyzer. We also present an experimental evaluation that shows the practicality of out scheme.

To allow the Data analyzer to verify the correctness of computations performed by an untrusted Aggregator each user selects uniformly and at random a secret key $\mathrm{tk}_{i} \in \mathbb{Z}_{p}$. The key dealer distributes to each user $g_{1}^{a} \in \mathbb{G}_{1}$ and publishes $g_{2}^{a} \in \mathbb{G}_{2}$, which calls for two exponentiations: one in $\mathbb{G}_{1}$ and one in $\mathbb{G}_{2}$. At each time interval $t$ each user computes $\sigma_{i, t}=H(t)^{\mathrm{t} \mathbf{k}_{i}}\left(g_{1}^{a}\right)^{x_{i, t}} \in \mathbb{G}_{1}$, which entails two exponentiations and one multiplication in $\mathbb{G}_{1}$. For the computation of the $\sigma_{\mathrm{t}}$ the Aggregator is involved in $n-1$ multiplications in $\mathbb{G}_{1}: \prod_{i=1}^{n} \sigma_{\mathrm{i}, \mathrm{t}}$. Finally the data analyzer verifies by checking the equality: $e\left(\sigma_{\mathrm{t}}, g_{2}\right) \stackrel{?}{=} e\left(H(t), \mathrm{vk}_{1}\right) e\left(g_{1}^{\text {sum }}, \mathrm{vk}_{2}\right)$, which asks for three pairing evaluations, one hash in $\mathbb{G}_{1}$, one exponentiation in $\mathbb{G}_{1}$ and one multiplication in $\mathbb{G}_{T}$ (see table 1). The efficiency of PUDA stems from the constant time verification with respect to the size of the users. This is of crucial importance since the Data Analyzer may not own computational power.

We implemented the verification functionalities of PUDA with the Charm cryptographic framework [1, 2]. For pairing computations, it inherits the PBC [13] library which is also written in C. All of our benchmarks are executed on Intel Core i5 CPU M $560 @ 2.67 \mathrm{GHz} \times 4$ with $8 \mathrm{~GB}$ of memory, running Ubuntu 12.04 32bit. Charm uses 3 types of asymmetric pairings: MNT159, MNT201, MNT224. We run our benchmarks with these three different types of asymmetric pairings. The timings for all the underlying mathematical group operations are summarized in table 3 . There is a vast difference on the computation time of operations between $\mathbb{G}_{1}$ and $\mathbb{G}_{2}$ for all the different curves. The reason is the fact that the bit-length of elements in $\mathbb{G}_{2}$ is much larger than in $\mathbb{G}_{1}$. 


\begin{tabular}{|l|c|c|c|}
\hline \hline Operation Pairings & \multirow{2}{*}{ MNT159 } & MNT201 & MNT224 \\
\hline Tag & & & \\
\hline Verify & $\mathbf{1 . 2} \mathrm{ms}$ & $\mathbf{1 . 8} \mathrm{ms}$ & $\mathbf{2 . 2} \mathrm{ms}$ \\
\hline
\end{tabular}

Table 2: Computational cost of PUDA operations with respect to different pairings.

\begin{tabular}{|c|c|c|c|}
\hline \begin{tabular}{ll|} 
Op. & Curve \\
&
\end{tabular} & MNT159 & MNT201 & MNT224 \\
\hline HASH in $\mathbb{G}_{1}$ & 0.13 & 0.346 & $0.296 \mathrm{~ms}$ \\
\hline $\mathrm{HASH}$ in $\mathbb{G}_{2}$ & $25.667 \mathrm{~ms}$ & $41.628 \mathrm{~ms}$ & $48.305 \mathrm{~ms}$ \\
\hline MUL in $\mathbb{G}_{1}$ & $0.004 \mathrm{~ms}$ & $0.0006 \mathrm{~ms}$ & $0.006 \mathrm{~ms}$ \\
\hline MUL in $\mathbb{G}_{2}$ & $0.040 \mathrm{~ms}$ & $0.051 \mathrm{~ms}$ & $0.054 \mathrm{~ms}$ \\
\hline MUL in $\mathbb{G}_{T}$ & $0.012 \mathrm{~ms}$ & $0.015 \mathrm{~ms}$ & $0.016 \mathrm{~ms}$ \\
\hline EXP in $\mathbb{G}_{1}$ & $0.072 \mathrm{~ms}$ & $0.092 \mathrm{~ms}$ & $0.099 \mathrm{~ms}$ \\
\hline EXP in $\mathbb{G}_{2}$ & $\mathrm{~ms}$ & $0.757 \mathrm{~ms}$ & $0.784 \mathrm{~ms}$ \\
\hline \begin{tabular}{|l|} 
PAIR \\
\end{tabular} & $7.077 \mathrm{~ms}$ & $10.674 \mathrm{~ms}$ & $13.105 \mathrm{~ms}$ \\
\hline
\end{tabular}

Table 3: Average computation overhead of the underlying mathematical group operations for different type of curves.

As shown in table 2, the computation of tags $\sigma_{i, t}$ implies a computation overhead at a scale of milliseconds with a gradual increase as the bit size of the underlying elliptic curve increases. The data analyzer is involved in pairing evaluations and computations at the target group independent of the size of the data-users.

\section{Related Work}

In [6], authors proposed a solution which is based on homomorphic message authenticators in order to verify the computation of generic functions on outsourced data. Each data input is authenticated with an authentication tag. A composition of the tags is computed by the cloud in order to verify the correctness of the output of a program $P$. Thanks to the homomorphic properties of the tags the user can verify the correctness of the program. The main drawback of the solution is that the user in order to verify the correctness of the computation has to be involved in computations that take exactly the same time as the computation of the function $f$. Backes et al. [3] proposed a generic solution for efficient verification of bounded degree polynomials in time less than the evaluation of $f$. The solution is based on closed form efficient pseudorandom function $P R F$. Contrary to our solution both solutions do not provide individual privacy and they are not designed for a multi-user scenario.

Catalano et al. [8] employed a nifty technique to allow single users to verify computations on encrypted data. The idea is to re-randomize the ciphertext and sign it with a homomorphic signature. Computations then are performed on the randomized ciphertext and the original one. However the aggregate value is not allowed to be learnt in cleartext by the untrusted aggregator since the protocols are geared for cloud based scenarios.

In the multi-user setting, Choi et al. [9] proposed a protocol in which multiple users are outsourcing their inputs to an untrusted server along with the definition of a functionality $f$. The server computes the result in a privacy preserving manner without learning the result and the computation is verified by a user that has contributed to the function input. The users are forced to operate in a non-interactive model, whereby they cannot communicate with each other. The underlying machinery entails a novel proxy based oblivious transfer protocol, which along with a fully homomorphic scheme and garbled circuits allows for verifiability and privacy. However, the need of fully homo- 
morphic encryption and garbled circuits renders the solution impractical for a real world scenario.

\section{Concluding Remarks}

In this paper we designed and analyzed a protocol for private and unforgeable aggregation. First we modeled its security and privacy requirements. In this setting a set of trustworthy users submit data coupled with unforgeable tags. The purpose of the protocol is to allow a data analyzer to verify the correctness of computation performed by a malicious Aggregator, without discovering the underlying data. The challenge of the verification in aggregation protocols that we tackled with the PUDA protocol is the fact that the privacy from the authentication tags is guaranteed by multiple independent users. Our PUDA instantiation allows for public verification in constant time and is provably secure under the DDH, BCDH and the new LEOM assumption in bilinear pairing groups in the random oracle model.

\section{Bibliography}

[1] J. A. Akinyele, M. Green, and A. D. Rubin. Charm: A tool for rapid cryptographic prototyping. http: / / www. charm-crypto.com/Main.html.

[2] J. A. Akinyele, M. Green, and A. D. Rubin. Charm: A framework for rapidly prototyping cryptosystems. IACR Cryptology ePrint Archive, 2011:617, 2011. http://eprint.iacr.org/2011/617.pdf.

[3] M. Backes, D. Fiore, and R. M. Reischuk. Verifiable delegation of computation on outsourced data. In ACM Conference on Computer and Communications Security, pages 863-874, 2013.

[4] D. Boneh, C. Gentry, B. Lynn, and H. Shacham. Aggregate and verifiably encrypted signatures from bilinear maps. In EUROCRYPT, pages 416-432, 2003.

[5] J. Camenisch and A. Lysyanskaya. Signature schemes and anonymous credentials from bilinear maps. In Advances in Cryptology - CRYPTO 2004, 24th Annual International CryptologyConference, Santa Barbara, California, USA, August 1519, 2004, Proceedings, pages 56-72, 2004.

[6] D. Catalano and D. Fiore. Practical homomorphic macs for arithmetic circuits. In EUROCRYPT, pages 336-352, 2013.

[7] D. Catalano, D. Fiore, and B. Warinschi. Homomorphic signatures with efficient verification for polynomial functions. In Advances in Cryptology-CRYPTO 2014, pages 371-389. Springer Berlin Heidelberg, 2014.

[8] D. Catalano, A. Marcedone, and O. Puglisi. Authenticating computation on groups: New homomorphic primitives and applications. In Advances in Cryptology - ASIACRYPT 2014 - 20th International Conference on the Theory and Application of Cryptology and Information Security, Kaoshiung, Taiwan, R.O.C., December 7-11, 2014, Proceedings, Part II, pages 193-212, 2014.

[9] S. G. Choi, J. Katz, R. Kumaresan, and C. Cid. Multi-client non-interactive verifiable computation. In Proceedings of the 10th Theory of Cryptography Conference on Theory of Cryptography, TCC'13, pages 499-518, Berlin, Heidelberg, 2013. Springer-Verlag. 
[10] D. M. Freeman. Improved security for linearly homomorphic signatures: A generic framework. In Public Key Cryptography - PKC 2012 - 15th International Conference on Practice and Theory in Public Key Cryptography, Darmstadt, Germany, May 21-23, 2012. Proceedings, pages 697-714, 2012.

[11] M. Joye and B. Libert. A scalable scheme for privacy-preserving aggregation of time-series data. In Financial Cryptography, 2013.

[12] I. Leontiadis, K. Elkhiyaoui, and R. Molva. Private and dynamic time-series data aggregation with trust relaxation. In Cryptology and Network Security - 13th International Conference, CANS 2014, Heraklion, Crete, Greece, October 22-24, 2014. Proceedings, pages 305-320, 2014.

[13] B. Lynn. The stanford pairing based crypto library. http://crypto. stanford.edu/pbc.

[14] A. Lysyanskaya, R. Rivest, A. Sahai, and S. Wolf. Pseudonym systems. In H. Heys and C. Adams, editors, Selected Areas in Cryptography, volume 1758 of Lecture Notes in Computer Science, pages 184-199. Springer Berlin Heidelberg, 2000.

[15] E. Shi, T.-H. H. Chan, E. G. Rieffel, R. Chow, and D. Song. Privacy-preserving aggregation of time-series data. In NDSS, 2011.

[16] V. Shoup. Lower bounds for discrete logarithms and related problems. In $A d$ vances in Cryptology - EUROCRYPT '97, International Conference on the Theory and Application of Cryptographic Techniques, Konstanz, Germany, May 11-15, 1997, Proceeding, pages 256-266, 1997.

\section{A Security evidence for the LEOM Assumption}

In this section we provide security evidence for the hardness of the new LEOM assumption by presenting bounds on the success probabilities of an adversary $\mathcal{A}$, which presumably breaks the assumption. We follow the theoretical generic group model (GGM) as presented in [16]. Namely under the GGM framework an adversary $\mathcal{A}$ has access to a black box that conceptualizes the underlying mathematical group $\mathbb{G}$ in which the assumption takes place. $\mathcal{A}$ without knowing any details about the underlying group apart from its order $p$ is asking for encodings of its choice and the black box replies through a random encoding function $\xi$ that maps elements from $\mathbb{G} \rightarrow \Xi$ as random bit strings of size $\left\lceil\log _{2} p\right\rceil$. Since our construction operates on asymmetric bilinear pairing groups $\mathbb{G}_{1}, \mathbb{G}_{2}, \mathbb{G}_{T}$ we make use of three random encoding functions $\xi_{c}, c \in[1,2, T]$ where $\xi_{c}: \mathbb{G}_{c} \rightarrow\{0,1\}^{\left\lceil\log _{2} p\right\rceil}$.

Theorem 5. Suppose $\mathcal{A}$ is a polynomial probabilistic time adversary that solves the LEOM assumption, making at most $q_{G}$ oracle queries for the underlying group operations on $\mathbb{G}_{1}, \mathbb{G}_{2}, \mathbb{G}_{T}$ and the $\mathcal{O}_{\mathrm{LEOM}}$ oracle, all counted together. All the encodings $\xi_{c}, c \in[1,2, T]$ and $\delta,\left\{\gamma_{u}\right\}_{u=1}^{n} \in \mathbb{Z}_{p}$ are chosen at random. Then the probability $\epsilon_{2}$ that $\mathcal{A}$ on input $\left(p, \xi_{1}(1), \xi_{2}(1), \xi_{1}(a), \xi_{1}(b), \xi_{1}(c), \xi_{2}(\delta), \xi_{2}\left(\sum_{i=1}^{n} \gamma_{i}\right)\right)$ to output a tuple $\left(\xi_{1}(a), \xi_{1}(b), \xi_{1}\left(c_{f}=\xi_{1}\left(\beta_{t} \sum_{u=1}^{n} \gamma_{u}+\alpha \delta \sum_{u=1}^{n} x_{u, t}\right)\right)\right)$ for which neither $x_{u^{\prime}, t^{\prime}} \neq x_{u, t}$ nor $\mathcal{A}$ has made more than $n$ distinct queries for a fixed time interval $t$, is bounded as:

$$
\epsilon_{2} \leq \frac{\left(q_{G}+16\right)^{2}}{p}
$$


Proof. We assume a polynomial time simulator $\mathcal{B}$ that interacts with adversary $\mathcal{A}$ and simulates the black box for the underlying groups $\mathbb{G}_{1}, \mathbb{G}_{2}, \mathbb{G}_{T} \cdot \mathcal{B}$ maintains 3 lists of tuples:

$$
\begin{aligned}
& -\mathcal{L}_{1}=\left\{\left(F_{1, i}, \xi_{1, i}\right): i=1, \cdots, \tau_{1}\right\} \\
& \text { - } \mathcal{L}_{2}=\left\{\left(F_{2, i}, \xi_{2, i}\right): i=1, \cdots, \tau_{2}\right\} \\
& \text { - } \mathcal{L}_{T}=\left\{\left(F_{T, i}, \xi_{T, i}\right): i=1, \cdots, \tau_{T}\right\}
\end{aligned}
$$

where $\quad F_{1, i} \in \mathbb{Z}_{p}\left[A, B,\left\{\Gamma_{u}\right\}_{u=1}^{n}, \Delta, X\right], F_{2, i}, \mathbb{Z}_{p}[\Delta, E] \quad$ and $\quad F_{T, i} \quad \in$ $\mathbb{Z}_{p}\left[A, B,\left\{\Gamma_{u}\right\}_{u=1}^{n}, \Delta, E, X\right]$ are multivariate polynomial on the indeterminants $A, B,\left\{\Gamma_{u}\right\}_{u=1}^{n}, \Delta, E, X$. Hereafter we will denote inteterminants for polynomials with capital letters and coefficients with lowercase. The random encodings $\xi_{c, i}, c \in[1,2, T]$ of each list $\mathcal{L}_{c}, c \in[1,2, T]$ are provided to the adversary $\mathcal{A}$ at each step $\tau$, where $\tau=\tau_{1}+\tau_{2}+\tau_{T}+4$. The lists are initialized at step $\tau=0$ by setting $\tau_{1}=1, \tau_{2}=3, \tau_{T}=0$ and assigning $F_{1,1}=1, F_{2,1}=1, F_{2,2}=\sum_{u=1}^{n} \Gamma_{u}, F_{2,3}=\Delta$, that corresponds to the generators $g_{1}, g_{2}$ and the public information $g_{2}^{\sum_{u=1}^{n} \gamma_{u}}, g_{2}^{\delta}$. $\mathcal{A}$ has access to the random encodings $\xi_{1,1}, \xi_{2,1}, \xi_{2,2}, \xi_{2,3}$ respectively.

In what follows we describe how $\mathcal{B}$ simulates the groups operations in $\mathbb{G}_{1}, \mathbb{G}_{2}, \mathbb{G}_{T}$ and the oracle responses to $\mathcal{O}_{\text {LEOM }}$. We first assume that before $\mathcal{A}$ queries the oracle or asks for group operations it has already asked for the random encodings of the elements involved in the operations. Consequently when $\mathcal{A}$ asks for operations in $\mathbb{G}_{c}, c \in[1,2, T]$ for some operands $\xi_{c}, c \in[1,2, T], \mathcal{B}$ checks if $\xi_{c}, c \in[1,2, T]$ already exists in $\mathcal{L}_{c}, c \in$ $[1,2, T]$ and aborts if this happens.

- Group operations: $\mathcal{A}$ provides $\mathcal{B}$ two operands $\xi_{c, 1}, \xi_{c, 2}, c \in[1,2, T]$ and a bit defining multiplication or division. $\mathcal{B}$ starts by incrementing $\tau_{c}+=1, c \in[1,2, T]$. It the computes $F_{1, \tau_{c}}=F_{1, i}+F_{1, j}$, where $1 \leq i, j \leq \tau_{c}$ if the operation bit is for multiplication or $F_{c, \tau_{c}}=F_{1, i}-F_{1, j}$, where $1 \leq i, j \leq \tau_{c}$ if it is for division. If the new polynomial $F_{c, \tau_{c}}$ is equal to another polynomial $F_{c, l}$ for some $l \leq \tau_{c}, c \in[1,2, T]$ in list $\mathcal{L}_{c}, c \in[1,2, T]$ then $\mathcal{B}$ fetches the corresponding $\xi_{c, l}$ and forwards it to $\mathcal{A}$, otherwise it chooses a fresh random $\xi_{c, \tau_{c}} \in\{0,1\}^{\log _{2} p}$ and gives it to $\mathcal{A}$. $\mathcal{B}$ finally appends to $\mathcal{L}_{c}, c \in[1,2, T]$ the pair $\left(F_{c, \tau_{c}}, \xi_{c, \tau_{c}}\right), c \in[1,2, T]$.

- Pairing: A pairing operation in $\mathbb{G}_{T}$ consists of two random encodings $\xi_{1, i}, \xi_{2, j}$ with $1 \leq i \leq \tau_{1}$ and $1 \leq j \leq \tau_{2}$. $\mathcal{B}$ first increments the counter $\tau_{T}+=1$. Afterwards it computes the pairing as the multiplication of the appropriate polynomials: $F_{T, \tau_{T}}=$ $F_{1, \tau_{1}} \cdot F_{2, \tau_{2}}$. If the same polynomial already exists in $\mathcal{L}_{T}: F_{T, \tau_{T}}=F_{T, l}, 1 \leq l \leq \tau_{T}$ then $\mathcal{B}$ clones the random string $\xi_{T, l}$, otherwise it chooses a fresh random $\xi_{T, \tau_{T}} \in$ $\{0,1\}^{\log _{2} p}$ and gives it to $\mathcal{A}$. $\mathcal{B}$ finally appends to $\mathcal{L}_{T}$ the pair $\left(F_{T, \tau_{T}}, \xi_{T, \tau_{T}}\right)$.

- $\mathcal{O}_{\text {LEOM }}: \mathcal{B}$ increments a counter $\tau_{\mathcal{O}}$ by 1 and sets $\tau_{1}+=3$. $\mathcal{A}$ inputs $\left(u, t, x_{u, t}\right)$. $\mathcal{B}$ computes the polynomials $F_{1, \tau_{1-2}}=A_{t}, F_{1, \tau_{1-1}}=A_{t}(Y), F_{1, \tau_{1}}=\left(B \Gamma_{u}+\right.$ $A \Delta X)$ for the indeterminants $B, \Gamma_{u}, A, \Delta, X$. If any of the $F_{1, \tau_{1-2}}, F_{1, \tau_{1-1}}, F_{1, \tau_{1}}$ already exists in $\mathcal{L}_{1}$ then $\mathcal{B}$ clones the associated random encodings $\xi_{1, l}$ for some $l \in\left[1, \cdots, \tau_{1}\right]$. Otherwise it creates three random encodings $\xi_{1, \tau_{1-2}}, \xi_{1, \tau_{1-1}}, \xi_{1, \tau_{1}} \in\{0,1\}^{\log _{2} p}$ and forwards them to $\mathcal{A}$. It also stores the pairs $\left(F_{1, \tau_{1-2}}, \xi_{1, \tau_{1-2}}\right),\left(F_{1, \tau_{1-1}}, \xi_{1, \tau_{1-1}}\right),\left(F_{1, \tau_{1}}, \xi_{1, \tau_{1}}\right)$ in $\mathcal{L}_{1}$ list. 
Eventually $\mathcal{A}$ outputs a forgery $\left(m_{f}, \xi_{1, f a}, \xi_{1, f y}, \xi_{1, f x y}\right)$. If $\mathcal{A}$ 's forgery is valid then it must hold:

$$
\frac{e\left(\prod c_{t}, g_{2}\right)}{e\left(\beta_{t}, g_{2}^{\sum_{u=1}^{n} \gamma_{u}}\right) e\left(a^{\sum_{u=1}^{n} m_{u}}, g_{2}^{\delta}\right)}=1 \in \mathbb{G}_{T}
$$

We show now that this does not happen always. Indeed w.l.o.g we have the following form for each polynomial in the three lists:

- $F_{1, i}=z_{0, i}+z_{1, i} h B \Gamma_{u, i}+z_{2, i} A \Delta X$, for coefficients $z_{0, i}, z_{1, i}, h, z_{2, i}$.

- $F_{2, i}=w_{0, i}+w_{1, i} \Delta+w_{2, i} E$, for coefficients $w_{0, i}, w_{1, i}, w_{2, i}$.

- $F_{T, i}=y_{0, i}+\eta_{1, i} \Delta h B \Gamma_{u, i}+\eta_{2, i} E h B \Gamma_{u, i}+\rho_{1, i} A \Delta^{2} X+\rho_{2, i} A \Delta X E$, for coefficients $y_{0, i}, \eta_{1, i}, h, \eta_{2, i}, \rho_{1, i}, \rho_{2, i}$.

Equation (1) following the aforementioned presentation of each polynomial can be rewritten as

$$
F_{f}=F_{T, k}-F_{T, l} F_{T, o}
$$

for indexes $k, l, o$. Simplifying the equation, since it is equal to 0 , then the second part consists of a polynomial with determinants $(\Delta \Gamma)^{2},(E \Gamma)^{2}, A \Delta^{4} X^{2},(A \Delta X E)^{2}$ and the first part with determinants $\left(\Delta \Gamma, E \Gamma, A \Delta^{2} X, A \Delta X E\right)$. Since there are no common terms, then all are canceled out and we are left with $y_{0, k}=y_{0, l} y_{0, o}$. As such $F_{f}=0$ only when $y_{0, k}=y_{0, l} y_{0, o}$.

$\mathcal{B}$ assigns random values $(\alpha, \beta, \gamma, \delta, e, x)$ for the indeterminants $A, B, \Gamma, \Delta, E, X$ and in order for $\mathcal{A}$ to win in the game, it should find two identical polynomial in any of the lists $\mathcal{L}_{1}, \mathcal{L}_{2}, \mathcal{L}_{T}$ or $F_{f}=0$. As such the success probability of $\mathcal{A}$ is bounded by the probability that one at least of the following equations holds:

1. $F_{1, i}(\alpha, \beta, \gamma, \delta, x)-F_{1, j}(\alpha, \beta, \gamma, \delta, x)=0: i \neq j$

2. $F_{2, i}(\delta, e)-F_{2, j}(\delta, e)=0: i \neq j$

3. $F_{T, i}(\alpha, \beta, \gamma, \delta, x)-F_{T, j}(\alpha, \beta, \gamma, \delta, x)=0: i \neq j$

4. $F_{f, i}(\alpha, \beta, \gamma, \delta, e, x)-F_{f, j}(\alpha, \beta, \gamma, \delta, e, x)=0: i \neq j$

$F_{1, i}$ degree is at most $3, F_{2, i}$ at most 1 , and $F_{T, i}$ at most 4 . . As such they vanish with probability $\frac{3}{p}, \frac{1}{p}, \frac{4}{p}$ respectively, from the Schwartz-Zippel theorem. As such summing for all possible pairs $i, j$ for each of the aforementioned polynomials the success probability of $\mathcal{A}$ is bounded by:

$$
\epsilon_{2} \leq\left(\begin{array}{c}
\tau_{1} \\
2
\end{array}\right) \frac{3}{p}+\left(\begin{array}{c}
\tau_{2} \\
2
\end{array}\right) \frac{1}{p}+\left(\begin{array}{c}
\tau_{T} \\
2
\end{array}\right) \frac{4}{p}+\frac{4}{p} \leq \frac{\left(\tau_{1}+\tau_{2}+\tau_{T}+12\right)^{2}}{p}
$$

As $\tau_{1}+\tau_{2}+\tau_{T} \leq q_{G}+4$ then $\epsilon_{2} \leq \frac{\left(q_{G}+16\right)^{2}}{p}$ 